This item was submitted to Loughborough's Research Repository by the author.

Items in Figshare are protected by copyright, with all rights reserved, unless otherwise indicated.

\title{
Monitoring 3D vibrations in structures using high resolution blurred imagery
}

PLEASE CITE THE PUBLISHED VERSION

http://dx.doi.org/10.1111/phor.12155

\section{PUBLISHER}

Wiley / @ The Remote Sensing and Photogrammetry Society and John Wiley \& Sons Ltd.

\section{VERSION}

AM (Accepted Manuscript)

\section{PUBLISHER STATEMENT}

This work is made available according to the conditions of the Creative Commons Attribution-NonCommercialNoDerivatives 4.0 International (CC BY-NC-ND 4.0) licence. Full details of this licence are available at: https://creativecommons.org/licenses/by-nc-nd/4.0/

\section{LICENCE}

CC BY-NC-ND 4.0

\section{REPOSITORY RECORD}

McCarthy, David, Jim Chandler, and Alessandro Palmeri. 2016. "Monitoring 3D Vibrations in Structures Using High Resolution Blurred Imagery". Loughborough University. https://hdl.handle.net/2134/21872. 


\title{
MONITORING 3D VIBRATIONS IN STRUCTURES USING HIGH RESOLUTION BLURRED IMAGERY
}

\author{
By DAVID M.J. MCCARTHY (d.mccarthy@lboro.ac.uk), \\ JIM H. CHANDLER (j.h.chandler@lboro.ac.uk) \\ and ALESSANDRO PALMERI (a.palmeri@lboro.ac.uk) \\ Loughborough University
}

\begin{abstract}
Photogrammetry has been used in the past to monitor the laboratory testing of civil engineering structures using multiple image based sensors. This has been successful, but detecting vibrations during dynamic structural tests has proved more challenging. Detecting vibrations during dynamic structural tests usually depend on high speed cameras, but these sensors often result in lower image resolutions and reduced accuracy.

To overcome this limitation, a novel approach described in this paper has been devised to take measurements from blurred images in long-exposure photos. The motion of the structure is captured in individual motion-blurred image, without dependence on imaging speed. A bespoke algorithm then determines each measurement point's motion. Using photogrammetric techniques, a model structure's motion with respect to different excitation frequencies is captured and its vibration envelope recreated in $3 D$. The approach is tested and used to identify changes in the model's vibration response.
\end{abstract}

KEYWORDS: Vibration, Deformation, Motion-blur, Engineering, Structural Health Monitoring, Close Range Photogrammetry

\section{INTRODUCTION}

CIVIL engineering structures often need to be tested to verify their performance and integrity. This requires meticulous planning, so that sufficient data is collected with the sufficient number of carefully positioned sensors. Contact transducers are often used, though they have some limitations, particularly if access to the structure is difficult. In the case of structural testing using dynamic test techniques, advanced sensors allow more detailed measurement. This approach is particularly convenient, as the presence of damage tends to result in a 
localised change in the stiffness within the structure, which can then affect the vibration response elsewhere.

The advantages of photogrammetry can be realised when applied to structural testing, as a very high number of points can be recorded with few sensors, without any contact with the structure. This is of primary relevance when measuring dynamic mode shapes, since interpolation is necessary between each measuring point. Current hardware, however, limits the potential for this type of dynamic monitoring, which necessitates much higher imaging speeds.

In this paper, a novel approach is developed to directly measure the blur created by a deliberately long exposure. This is explained and tested after the importance and prior work is reviewed. The approach has built on previous developments (McCarthy et al., 2013, 2014) and the results of enhanced case studies are included here.

\section{STRUCTURAL TESTING}

ASSESSMENTS of structural integrity are often necessary for a variety of reasons. New materials under development in the laboratory environment may demand complex monitoring schemes to understand deformation patterns. Testing may also be prescribed during construction as verification of some critical elements. Testing of existing structures may be needed for evaluation prior to a change of use, or following a potentially damaging event. Long term structural health monitoring schemes are necessary on some structures to detect any potential deterioration.

Conventional structural tests apply increasing load to a structure while its performance is monitored by gauges. Subject to acceptable results, the structure is then deemed suitable for its working loads. The load would be applied with, for example, test weights on a slab or large vehicles on a bridge. This testing of 'static' structural properties may be considered inconvenient since the test would necessitate taking the structure out of service, in addition to the logistical problems of transporting and moving loads. Care must also be taken to not increase load above the structure's elastic limit, otherwise damage may occur from the test itself.

Dynamic structural monitoring is now emerging as a viable and effective approach for assessing structural performance (Brownjohn, 2011). Data collection is considered more convenient since much lower loading is necessary and monitoring can take place without taking the structure out of service. However, data analysis can be complicated and specialist expertise is necessary to interpret the vibration response. Several approaches exist to identify structural changes from the measured vibration response (Kasinos et al., 2014) and comparative studies score these approaches on their ability to identify, locate and quantify damage (Carden \& Fanning, 2004). Generally, those approaches which take advantage of a higher number of monitoring points produce the most reliable results (Carden \& Fanning, 2004). 


\section{MONITORING OF STRUCTURAL TESTS}

ENGINEERS must select and distribute sensors appropriately to collect sufficient data to understand forces and deformations within a structure. Traditional contact sensors measure displacements or strains. For example, linear variable displacement transducer (LVDT) sensors can be used to measure displacement at various points of the structure, usually at a beam's mid-span or other equally spaced locations. Foil strain gauges measure strain in the material, the dimensionless measure of extension in a given direction.

Traditional contact gauges are reliable, well understood and often prescribed by engineers, but in many cases their effectiveness is limited by the fact that they only record the structure's performance in a single location, at the position where they are fitted. The possibility of individual gauges being affected by local variations in the material, or local strain concentrations should be recognised. Strain gauges are also susceptible to daily and seasonal environmental changes, being a significant limiting factor in long term structural health monitoring. Furthermore, each additional sensor costs money, takes time to fit and requires cabling infrastructure, and many sensors only measure in one dimension unless the more expensive multidimensional counterparts are used. Harvey (2008) noted the importance of carefully locating sensors, as a poorly sited sensor may not measure anything useful.

More advanced non-contact sensors can be used, including a total station or terrestrial laser scanner. These sensors can collect optical measurements from several monitoring points, but the latter is unable to measure true instantaneous point deformation. In addition, they take time to traverse the whole structure, making them suitable for testing of static properties only (Psimoulis and Stiros, 2008; Ronnholm et al., 2009). Differential GPS can monitor deformation without line of sight, but each individual monitoring point adds considerable cost (Roberts, 2004). The laser Doppler vibrometer (LDV) is a specialised optical dynamic sensor for monitoring vibrations. Changes in the wavelength of a reflected laser beam are related to the velocity of a moving surface. The instrument is very precise, but measures only at the location it is targeted at.

Photogrammetry has demonstrated its potential for monitoring structural tests in the past (Cooper, 1990; Maas, 1998; Benning, 2006). In particular, it allows instantaneous measurement of a very high number of monitoring points with a single arrangement of image sensors. These points may be monitored in $3 \mathrm{D}$ if using stereo or multiview imagery, whereas many contact gauges are one dimensional, or have more expensive 3D variants. Other advantages include that it is non-contact, meaning that the monitoring instruments do not add load to the test subject, and a stable reference on which to support contact gauges is not required. In particular, the photogrammetric approach is scalable, and suitable for a wide range of applications from small-scale laboratory tests (Thomas \& Cantré, 2009) to full-size structures.

Photogrammetry has also been shown suitable for monitoring dynamic tests. Since all monitoring points within an image are measured simultaneously, additional points can be added, and the distance between each monitoring point reduced, with little additional cost. Vibrations can be captured by increasing 
imaging speed to many frames per second (fps). The Nyquist sampling theorem dictates that, in order for certain frequencies to be detected, data must be sampled at least twice the desired frequency range. Olaszek (1999) notes that an even higher sampling frequency may be desirable, since even if the Nyquist theorem is satisfied, aliasing effects can cause the peak amplitude in the frequency domain to be missed.

Structural engineers always desire measurements that are as accurate as possible. Clearly the use of the highest resolution cameras is desirable to achieve the highest precision measurement but, as noted above, high imaging speed is normally necessary to capture and hence measure vibration at the required frequencies. When selecting camera hardware for monitoring tests of static properties (which take place over several minutes or a few hours), potential speed of continuous imaging may not need to be considered. In contrast, for dynamic tests with relatively fast variations in the deformed state of the structure, consideration must be given to both the image resolution and the potential continuous imaging speed of the camera.

When selecting a camera, current sensor hardware requires a compromise to be made between the image resolution and temporal resolution of the sensor. Digital single lens reflex (DSLR) cameras are available with image resolutions over $30 \mathrm{MP}$ and consumer camcorders typically record at up to 60 frames per second. However, a DSLR camera at its highest resolution setting can only sustain at most a few images per second. Consumer camcorders acquire images at higher frequencies, but only capture at much lower image resolutions, typically $2.1 \mathrm{MP}$ ('HD' resolution). Specialist machine vision camera sensors exist in a wide variety of configurations, but even these sensors are limited by a trade-off between spatial resolution and imaging frequency, and are expensive. Although the capabilities of image sensors are continually improving, so too do the demands of engineering researchers.

Real-time monitoring is very often desired for monitoring structural tests, since the engineer can appreciate the structure's performance as the tests takes place. When high speed imaging is used for monitoring, the images must be processed at the same rate that they are acquired, otherwise the real-time detectable vibration frequencies are limited by the processing speed. Real-time monitoring at acceptable speeds is possible, but only at a reduced image resolution and hence reduced spatial accuracy or by reducing the number of points (Jurjo, 2010), increasing undesirable interpolation.

Capturing a very high number of high resolution images also has practical difficulties. A considerable amount of data is generated and bottlenecks exist when images are stored to an internal memory card or uploaded to external file storage, or another computer for processing. If images are post-processed, or are to remain as documentary evidence, a large amount of data storage space is required. 


\section{PROPOSED MEASUREMENT APPROACH}

AIMED at alleviating this penalising compromise between image resolution and imaging frequency, a radically new approach is presented in his paper, in which the structural vibration is captured within long-exposure images. Indeed, the motion of the vibrating structure causes localised blur within the image, and this can be measured using automated image processing methods.

Motion blurred imagery has been examined in Computer Science literature, and many algorithms have been published for extracting data from these seemingly poor quality images (Yitzhaky \& Boshusha, 2000; Wang et al., 2007). It is often assumed that a motion blurred image is created from an ordinary sharp scene which is degraded my some blurring function. This blurring can be expressed using (Banham \& Katsaggelos, 1997):

$$
\mathbf{Y}(i, j)=\sum_{k=1}^{m} \sum_{l=1}^{n} \mathbf{H}(i, j ; k, l) \mathbf{F}(k, l)+\mathbf{N}(i, j)
$$

where, $\mathbf{F}(k, l)$ is an ordinary $m \times n$ sharp image; $\mathbf{H}(i, j ; k, l)$ is the point spread function; $\mathbf{Y}(i, j)$ is the resultant blurred image; and $\mathbf{N}(i, j)$ represents noise.

The point spread function, $\mathbf{H}$, contains information about the motion that caused the blur, including its extent, direction, and distribution. Some algorithms are capable of 'blind deconvolution' i.e. recovering the point spread function without prior knowledge of the motion that caused the blur.

In our implementation, an algorithm measures the motion blur exhibited by ordinary circular targets (McCarthy et al., 2014). Both the amplitude of the motion causing the motion blur and direction of the motion path are measured. A sinusoidal motion is assumed, as is expected during frequency selective dynamic structural testing (e.g. Chopra, 2007; Clough \& Penzien, 2010; Palmeri \& Lombardo, 2011). Black circular targets with a white background are arranged at necessary monitoring points on the test subject in the conventional way. Our implementation also requires a single sharp image of the test structure from the same cameras. This may be before dynamic excitation is applied, or in the case of a structure with environmental loading which vibrates continuously, simply an image taken with an appropriately fast shutter speed.

When the test structure vibrates, either artificially by manually applying force, or naturally under environmental loads such as wind, a single long exposure image is taken. As a result, the whole vibration of the structure during the time that the shutter is open is captured within the single image. The motion amplitude and direction at each monitoring point can then be measured by processing these long exposure images with a bespoke algorithm, implemented in MATLAB.

A set of preliminary tests were carried out on circular targets, which have demonstrated that their blurred images take a consistent form, exhibiting the same edges, shapes and gradients. Interestingly, two cases exist whereby the motion amplitude is either less or greater than the diameter of the circular target (Figure 1). In the former case, a small region of the image is occupied by the target throughout the exposure, and the pixels in this region remain the same 
intensity as the black sharp target exhibiting no motion. Other image pixels which are occupied by the target for only part of the whole exposure, generate a pixel value with a value between the black target and the white border, proportional to the amount of time occupied by the target. In the latter case, every region of the image is exposed to the white target border for at least some of the time that the shutter is open, so the pixel value measured by every pixel within the blurred target image is in between the black of the target and white of the target background.

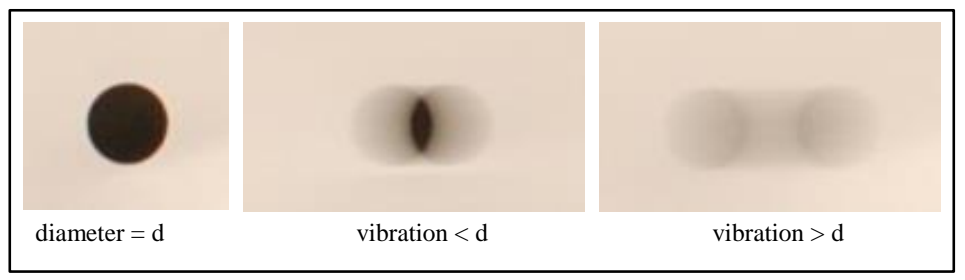

Figure 1: Appearance of motion blurred circular targets

\section{IMAGE PROCESSING PROCEDURE}

THE KEY steps in the bespoke image processing algorithm are summarised in Figure 2. The proposed procedure uses MATLAB's image processing toolbox. Three measurements are estimated using different techniques, each achieving increasing accuracy, but at decreasing speed.

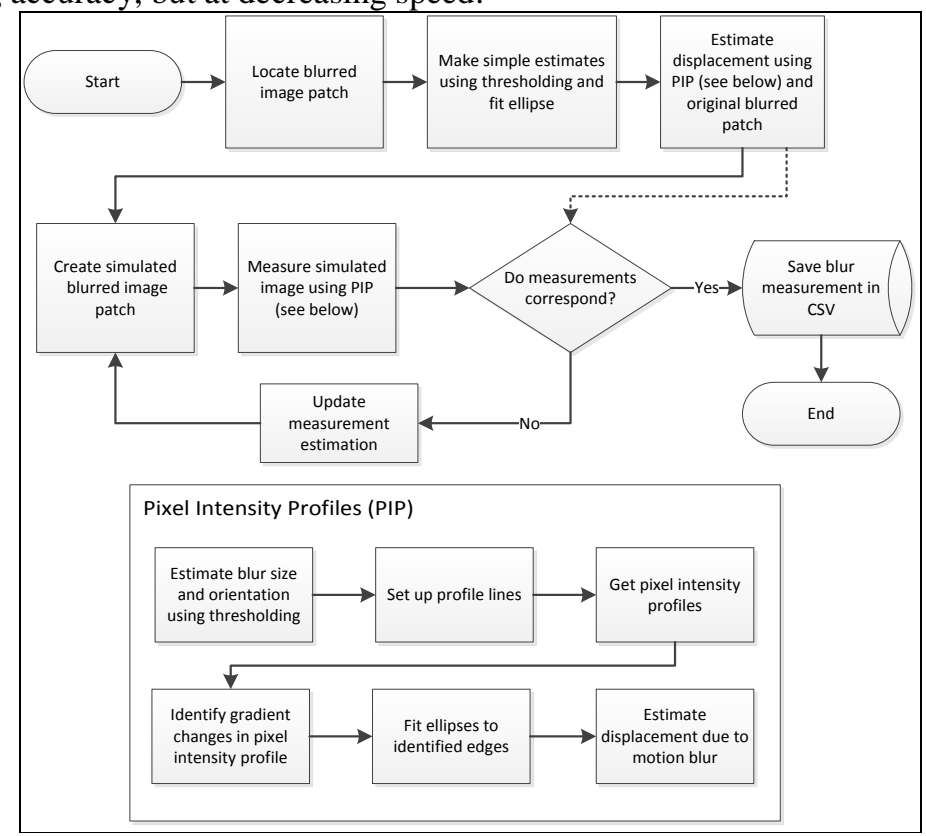

Figure 2: Blur measurement calculation flowchart 
The blur measurement algorithm assumes the approximate location of each target is already known, since blurred targets in an image are more difficult to locate than a sharply defined target. This is achieved by detecting targets in the additional sharp image, in which circular targets are easily identified, and a list of coordinates passed to the blur measurement algorithm.

After locating the image patch of the blurred target, a rapid approximate estimate about the geometry of the image is determined with a simple thresholding algorithm. The chosen threshold needs to be nearer in value to the white of the target background, so that the detected region includes the grey regions at the edge of the blurred target. An ellipse is fitted to the detected region using least squares, and the estimated major and minor axis, and orientation allows arrangement of the profile lines for the next stage (Figure 3).

\section{Figure 3: Major and minor axis (red and blue) of the target with arranged pixel intensity profile lines (green)}

Secondly, parallel Pixel Intensity Profiles (PIPs) are extracted from the image through the major axis of the blur. The PIP process is adapted from an approach proposed by Boracchi et al. (2007). The number of profiles can be adjusted, but 20 was found to be a suitable balance between measurement accuracy and processing time. An example pixel intensity profile appears as in Figure $4 \mathrm{~b}$ (The signal is inverted so that target pixels result in a peak), and points can be identified at abrupt changes in the gradient of the intensity profile which relate to features on the blur smear (Figure 4a). The algorithm identifies these features twice differentiating the intensity profile and locating peaks in the resulting function. These features are allocated to two groups (shown in red and blue in the figure), depending on where they follow or precede positive or negative gradients, and ellipses are fitted to each group of points (Figure 4c). The distance and angle between the centre coordinates of these ellipses is taken as the estimated motion occurring at that monitoring point on the structure. Tests demonstrated that this method provides pixel level accuracy of the motion amplitude.

The pixel intensity profile algorithm is effective in both cases, where motion amplitude is both smaller and larger than the target diameter (Figure 1); the algorithm identifies changes in gradient rather than changes in absolute pixel value. The type of blurred target is automatically identified by the presence of the dark centre region, since this has an effect on the allocation of feature points in the pixel intensity profiles into the two ellipses.

In tests, the pixel intensity profiles method alone regularly over-estimated the exhibited motion and did not provide adequate accuracy due to a specific systematic effect. Motion was overestimated because at the edge of targets there is 
a gradient in pixel values, caused by chromatic aberration and lens diffraction. This was evident when an image of a stationary target exhibited an apparent displacement of approximately 1 pixel. A subsequent step was included therefore to improve accuracy.

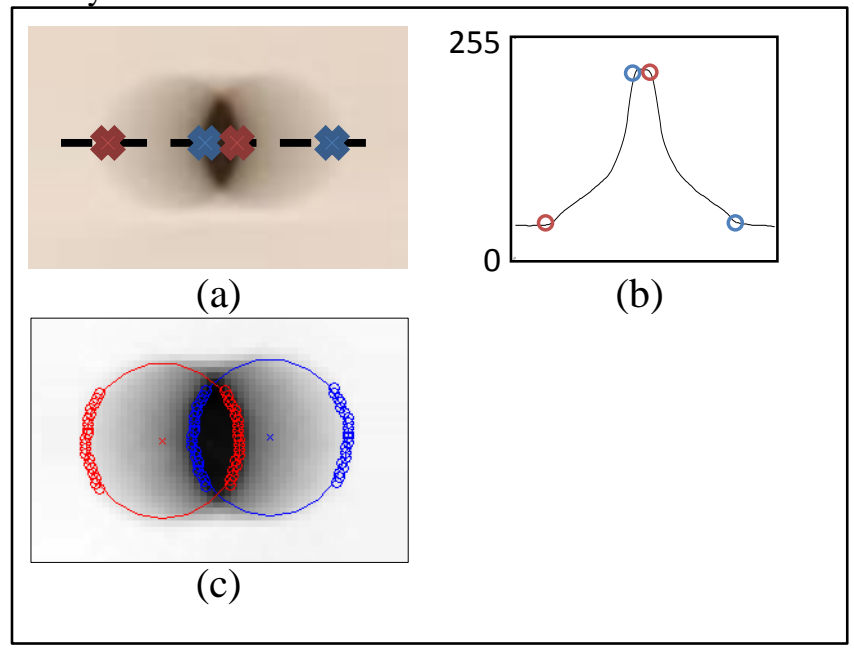

Figure 4: Resultant Blur Measurement

To achieve superior accuracy an iterative procedure was introduced. Here, a simulated blurred image patch is generated using the blur function (equation 1), using a sinusoidal point spread function of the first estimated motion and the earlier taken sharp image. The equation used to describe the point spread function, $\mathbf{H}$, is defined by:

$$
f(x)=\left\{\begin{array}{cc}
0, & x<\frac{L-1}{2} \\
\cos \frac{2 \pi x}{L}+1, & \frac{L-1}{2} \leq x \leq \frac{L+1}{2} ; \\
0, & x>\frac{L+1}{2}
\end{array}\right.
$$

where, $L$ is the estimated motion amplitude. This function is discretised into units of a pixel by applying the trapezium rule over pixel intervals. The result of this function is spatially rotated by the estimated motion angle using MATLAB's imrotate function set to use bilinear interpolation. Finally, the matrix is divided by the sum of its elements, so that the elements of the final $\mathbf{H}$ sum to unity.

Pixel intensity profiles of this simulated image are measured and compared with the as-taken blurred image. If the initial measurement was correct, the astaken and simulated image would correspond closely. If a discrepancy between the two remains, the amplitude and angle parameters of the point spread function are iteratively adjusted by the measurement discrepancy until the difference between the measured motion in both the original and simulated image patches is less than a user set threshold (Fig 5). The motion amplitude and angle used to create a simulated image that matched the as-taken image, is then accepted as the vector describing the distance and direction of the motion at that point on the test subject. 


\section{TRANSFORMATION INTO OBJECT SPACE}

Having measured image coordinates in units of pixels, the distance and direction can be immediately represented graphically by superimposing displacement vectors on to the original images. By also superimposing the envelope of the motion, the output is similar to that produced by finite element software packages that might be used to predict forces and deformations in the structure. By showing displacement vectors together with an image of the structure, the engineer can appreciate which parts of the structure have the greatest motion, as well as observing the distribution, and possibly spot any anomaly.
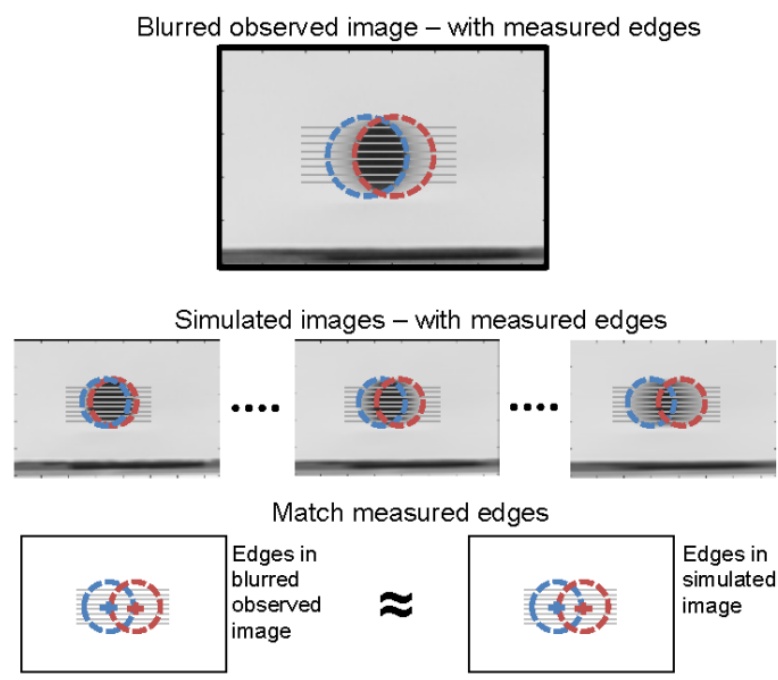

Figure 5: Iteratively improving accuracy using both the original blurred and simulated image patches.

\section{D Planar Measurement}

It is also likely that an engineer would desire these measurements transformed into an appropriate object-space system (in millimetres) for more precise comparisons and analysis. With only a single camera, it is possible to provide 2D measurements, so long as it is known that any movement is planar and the focal plane of the camera is aligned approximately to the same plane. In this scenario, the perspective transformation (Luhmann et al., 2006) can be used to transform coordinates from image space to object space. However, if any out-ofplane movement were to occur, this could cause a scaling error in the measured results. It is recognised that radial and tangential distortion cause a displacement of a projected object point in the image plane. However, if individual monitored points are displaced relative to a fixed camera, then computed object displacements are almost identical, whether a lens model is explicitly incorporated or not. However, the effects of lens distortion can be easily corrected following 
camera calibration (eg. Luhmann et al., 2006) and a correction for this simple systematic effect should be incorporated.

Coded control targets can simply be arranged around the test object (coplanar to the monitoring targets) and their object-space coordinates measured using a reflectorless Total Station. Coordinates in an arbitrary 3D object space are calculated. For calculating planar deformations, plane space coordinates for each control point are determined (Fig 6). The plane is defined by the object-space $X$ and $Y$ coordinates, to which linear regression is used to define an object space plan gradient and intercept. The distance from the intercept to the nearest point along the regression line of each control point is taken as its plane-space $x$ coordinate. The earlier determined object-space $Z$ coordinates become corresponding planespace vertical $y$ coordinate. Projective transformation parameters are determined with the paired plane-space and image-space control target coordinates, which are used to apply the projective transform to the earlier measured image-space monitoring point coordinates.



Figure 6: Definition of planar coordinates from 3D control point coordinates

\section{Measurement in 3D}

3D measurement has been highlighted in the past as a strength of photogrammetric measurement for monitoring of structures. The monitoring approach was expanded to fully 3D measurement that also allows non-planar motion to be recorded (McCarthy et al., 2014). For a moving target, two 3D object points will be created on any image, representing the target at each end of its motion. A 3D approach requires the use of two or more sensors and their shutters must be interconnected so that they capture images simultaneously. Additional calculation steps for finding 3D coordinates are shown in figure 7.

The exterior orientation (EO) of the cameras needs to be known and was achieved using the PhotoModeler software package (version 2013.0.3.113 64-bit, July 2013; EOS Systems Inc., Vancouver, Canada). This software is convenient because it automatically recognises coded control targets in images, and object space coordinates for the control points can be loaded using a PhotoModeler coded coordinate file. PhotoModeler's automated camera calibration was also used to determine appropriate interior orientation (IO) parameters, including 
deriving an appropriate lens model, allowing accurate coordinates for the monitoring points to be determined. These object points allow approximate image coordinates to be computed, which are then used by the blur measurement algorithm to assist locating targets in images. Target matching can also be carried out by PhotoModeler.

Image coordinates for the motion blurred targets are measured as before using the image processing algorithm described above and stored in a CSV file. As mentioned above, a new challenge at the point matching stage occurs, since each target, which would normally have a single measurement in each image, instead has two coordinates measured, at either end of its motion path. Unlike traditional photogrammetry, the image coordinates relating to a $3 \mathrm{D}$ point cannot be uniquely matched with coded targets since they originate from one physical target which changes position. Figure 8 shows how two image coordinates exist for each target, and how different pairs of candidate object points can be created.

To solve this ambiguity for all objects/camera configurations, two candidate combinations of the pairs of coordinates for each target should be considered, since it is not yet known which combination is correct. 3D coordinates are therefore calculated for both combinations using a bespoke space intersection program, using Photomodeler's earlier derived EO and IO, which explicitly corrects for lens distortion. Residual image coordinates for the incorrect combination are usually significantly higher, depending on the exact arrangement (although, where the direction of target motion approaches the epipolar plane, they become similar, as discussed below). Residual coordinates are then calculated through reprojection of the $3 \mathrm{D}$ point onto the image, using the known camera orientations. In tests, it was found that selecting the combination of image coordinates which produces the smallest residual measurements overall, usually identified the correctly matched pair.

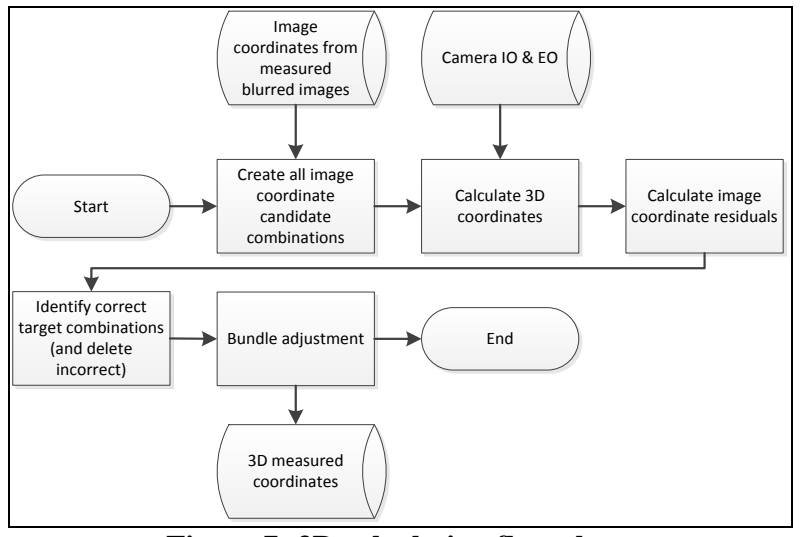

Figure 7: 3D calculation flow chart

Although this method has proven successful, in a particular case the correct combination cannot be automatically determined; where the orientation of the both measured points lie on the same epipolar plane. It may be possible to determine the most likely combination using engineering judgement, but if "a priori" 
knowledge of the likely motion is available, then the two cameras can be arranged obliquely, such that this situation does not occur. It is therefore helpful to consider likely target movement direction when selecting camera positions. This ambiguity could also be solved by adding a third camera to the system, although there would then be $2^{3}=8$ combination of coordinates to consider for a single target.

Once 3D coordinates are determined, all of this data were then input to a bundle adjustment ('General Adjustment Program' (GAP), Chander \& Clarke, 1992) to calculate final 3D coordinates in the object space.

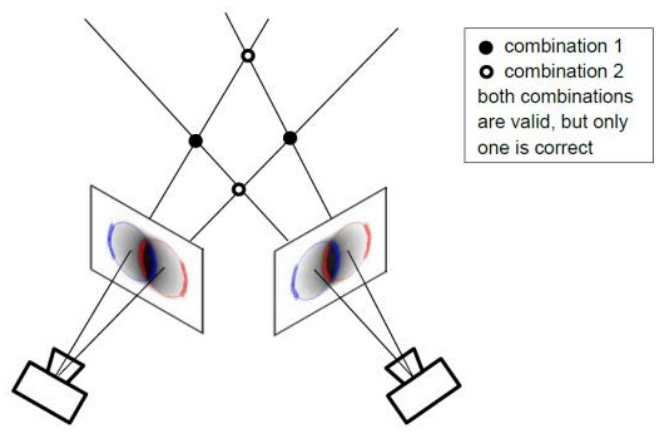

Figure 8: Point matching difficulties for a single target

\section{PRACTICAL TESTS AND RESUlTS}

A ONE dimensional shaker table was used to test and demonstrate the proposed approach. The APS 'Electro-Seis' 400 is a long-stroke shaker capable of carrying out programmed one-dimensional motions up to $150 \mathrm{~mm}$ described in McCarthy et al. $(2013,2014)$. In its normal use, models are fitted to the $455 \mathrm{x}$ $455 \mathrm{~mm}$ table bed which is programmed with vibrations of varying frequencies and amplitudes. As the model is subjected to base vibration, data would be recorded about the model's dynamic response using accelerometers and a laser displacement gauge.

Two Nikon D80 DSLR cameras were available for image capture, each camera equipped with $24 \mathrm{~mm}$ fixed focal length lenses and focus fixed at the appropriate distance (Figure 9a). The cameras have a sensor resolution of 10.2 MP. The shutter speed was fixed at 1 second in order to capture the whole vibration cycle, and the aperture and sensitivity were set to F32 and ISO 100 to provide clear images with appropriate contrast. The external shutter release for each camera was connected to the shaker table's control system (Figure 9c) so that the exact time they are triggered is recorded alongside the sensor data so that a direct comparison can be made.

Simple plastic model structures with a height of $760 \mathrm{~mm}$ were rigidly fixed with bolts to the shaker table (Figure 9b). The columns on the structure were marked with circular targets. Black $8 \mathrm{~mm}$ circles were printed on white paper backing with sufficient white space around the target so that the white space was sure to exceed the expected motion amplitude. Conventional coded targets were 
arranged around the shake table to provide control points, and their positions were measured by total station in reflectorless mode. For 2D monitoring, these must be in the same plane as the monitoring targets, whilst for 3D tests these define a volume occupied by the model structure.

\section{Testing 2D monitoring}

For the first test, 2D measurements were made with a single camera positioned in front of the structure. The model structure was subjected to one dimensional

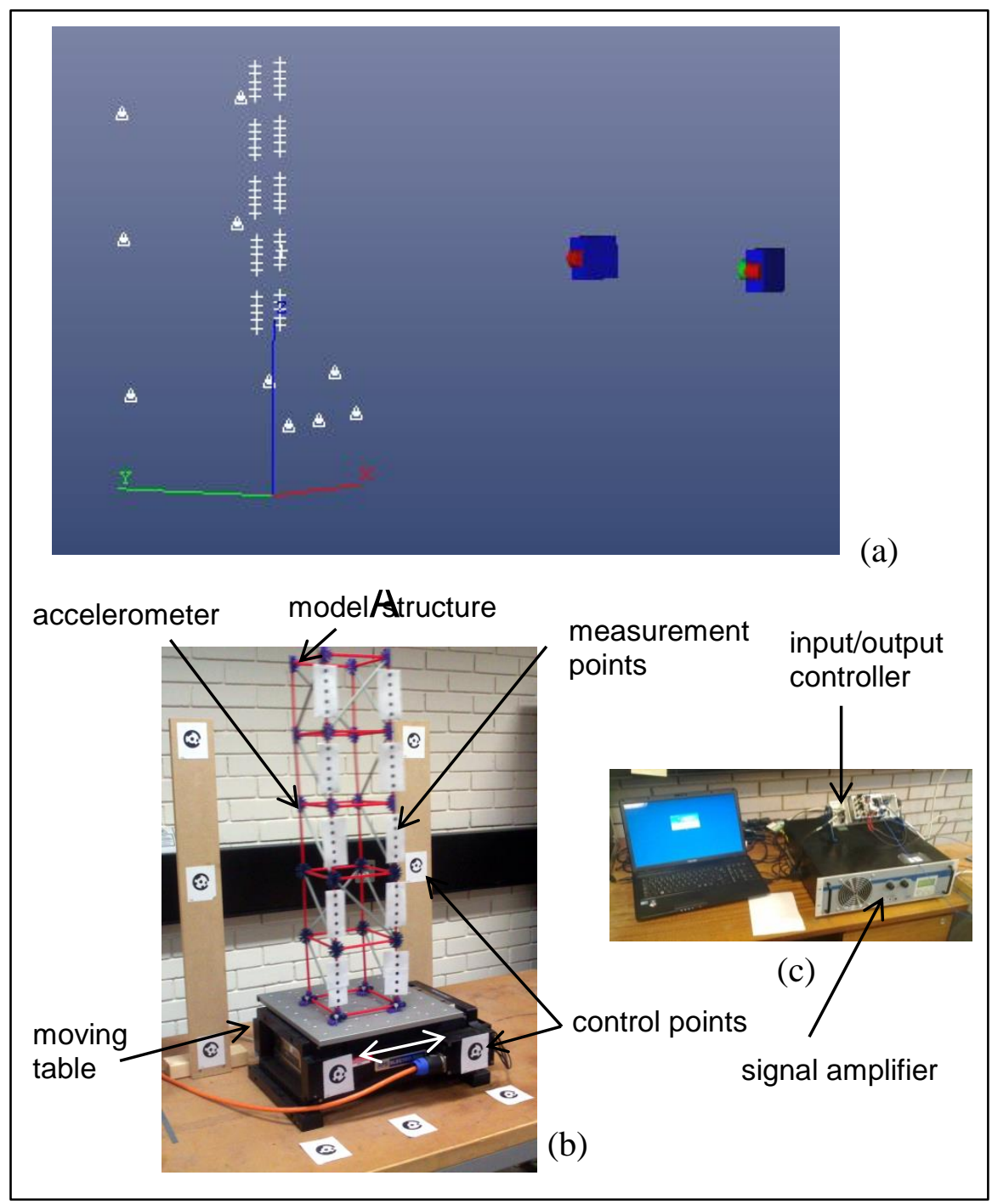

Figure 9: (a) experimental set up, (b) detail of vibration test subject, (c) detail of shaker table control system. 
motion of varying frequencies. In particular, the model was excited with sinusoidal vibration at its natural frequencies (established separately using an accelerometer with a 'sine sweep' input table motion) so that the model would exhibit its vibration envelope for these modal frequencies. These are of interest for looking at the curvature of the vibration envelope, and locating nodes and antinodes (Abdel Wahab, 1999). Figure 10 shows recorded images overlain with the $2 \mathrm{D}$ measurements established using the image processing routine described in the previous section ("2D Planar Measurement").

The shape of the first vibration mode at $5 \mathrm{~Hz}$ may be expected for a structure of this type. The higher vibration mode shapes are less predictable since they are more sensitive to local variations in the structural properties. These higher modes are therefore also more likely to be able to indicate any changes in stiffness caused by damage or degradation of a structure.

\section{Testing 3D Monitoring}

Taking advantage of appropriate photogrammetric procedures and processes, further tests were carried out of the same model to demonstrate the 3D deformation measurement system developed. The second camera was introduced, and cameras rearranged to achieve appropriately convergent images. Both camera's external triggers were connected to the shaker table's control system, so that they would both be triggered simultaneously. The same motion was reapplied to the model. Following data capture, the images were processed as before using the blur measurement algorithm. The steps described in the previous section "Measurement in 3D" for determining the camera's orientations were followed, and $3 \mathrm{D}$ coordinates generated. Figure 11a shows the results from the repeated test

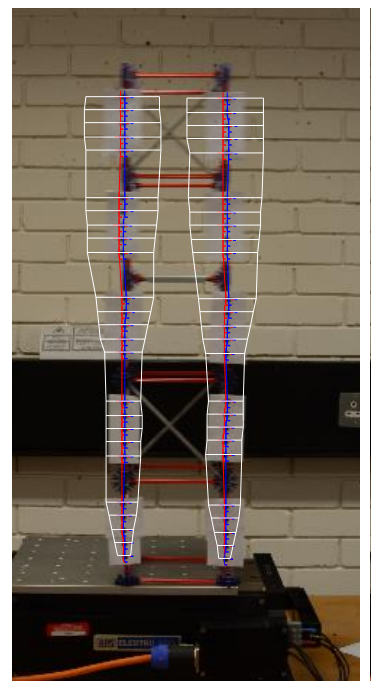

1st mode $(5 \mathrm{~Hz})$

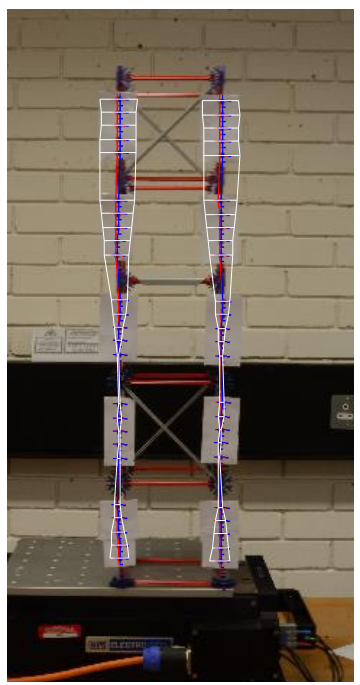

2nd mode $(8 \mathrm{~Hz})$

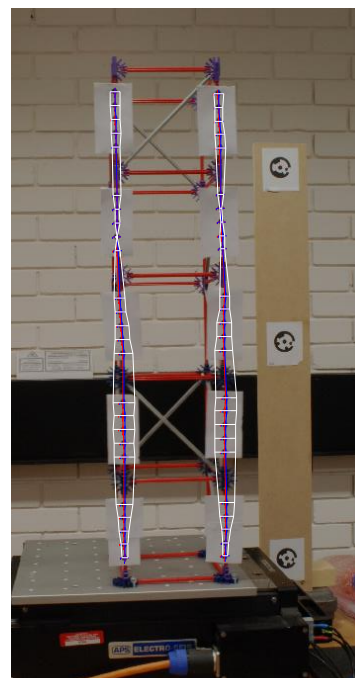

3rd mode $(12 \mathrm{~Hz})$

Figure 10: Measured vibration envelopes (horiz. scale enlarged by factor of 20) 
which are similar in form to those measured in the first test (Figure 10).

\section{Detecting structural changes}

To assess the potential for detecting structural changes using the measured image data, a further test was designed using the same model structure. As shown in Figure 12, the structure was modified by adding mass to the structure on the $4^{\text {th }}$ level. The test was repeated with the first three mode shapes being captured. Figure $11 \mathrm{~b}$ shows the change in the dynamic response of the modified structure can be appreciated by a change in the measured vibration envelope. In particular, more significant three-dimensional changes are observed at the higher modes.

The second modification involved substituting two vertical members with replacements that had part of their cross section cut away (figure 12). By reducing

\begin{tabular}{|c|c|c|c|}
\hline & 1st mode $(5 \mathrm{~Hz})$ & 2nd mode $(8 \mathrm{~Hz})$ & 3rd mode $(12 \mathrm{~Hz})$ \\
\hline 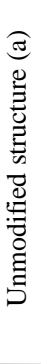 & 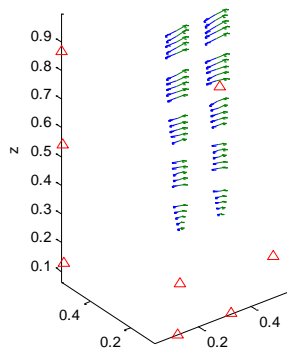 & 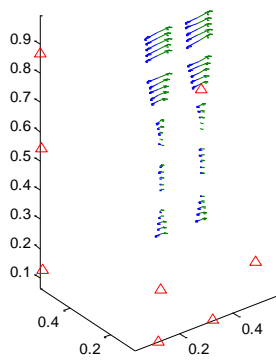 & 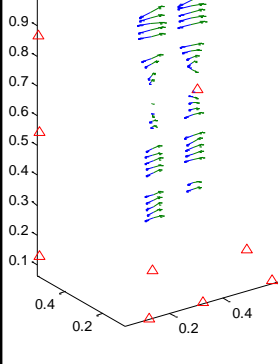 \\
\hline 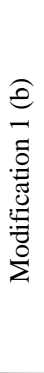 & 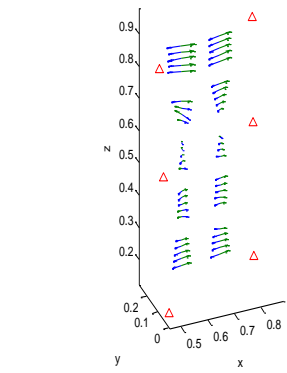 & 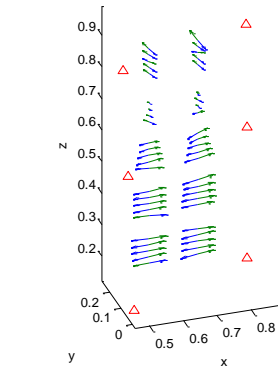 & 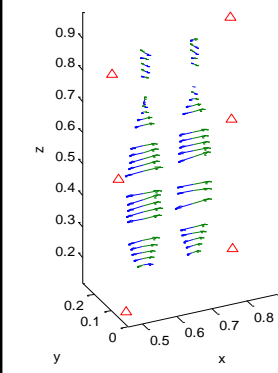 \\
\hline & 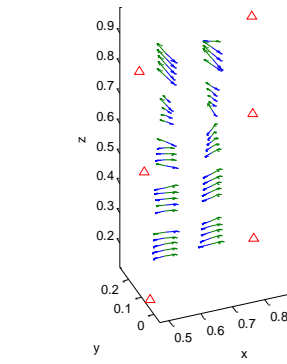 & 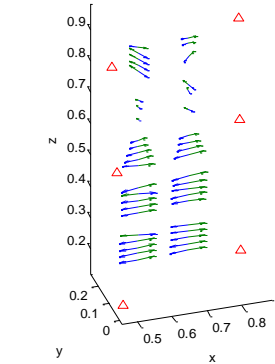 & 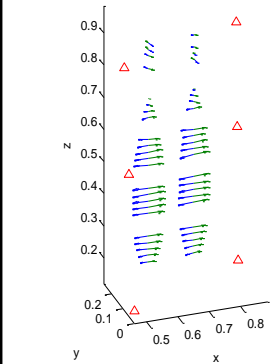 \\
\hline
\end{tabular}

Figure 11: Measured change in vibration envelope from structural modifications. Blue/green vectors represent individual target displacements, whilst red triangles represent control points. 
the cross-sectional area of the member, the stiffness was reduced. This is a similar affect to the failure of welds in a steel structure, which would result in a loss of stiffness. Changes can be observed in the locations of nodes and anti-nodes, the positions where the amplitude is smallest and largest. In a practical setting, identifying a change in the vibration envelope when one was not expected would prompt further investigation as to the underlying cause, possibly prompting repair work (Kasinos et al., 2014).

Testing was repeated again and results are presented in Figure 11c. It is interesting to note how the analysis of the unmodified model (Figure 11a) reveals a motion which is essentially planar in all the three modes of vibration; while the two modifications (Figures $11 \mathrm{~b}$ and 11c) induces some significant torsional effects. Such changes in orientation of the vibration envelope may not ordinarily be detectable with conventional single axis accelerometers.

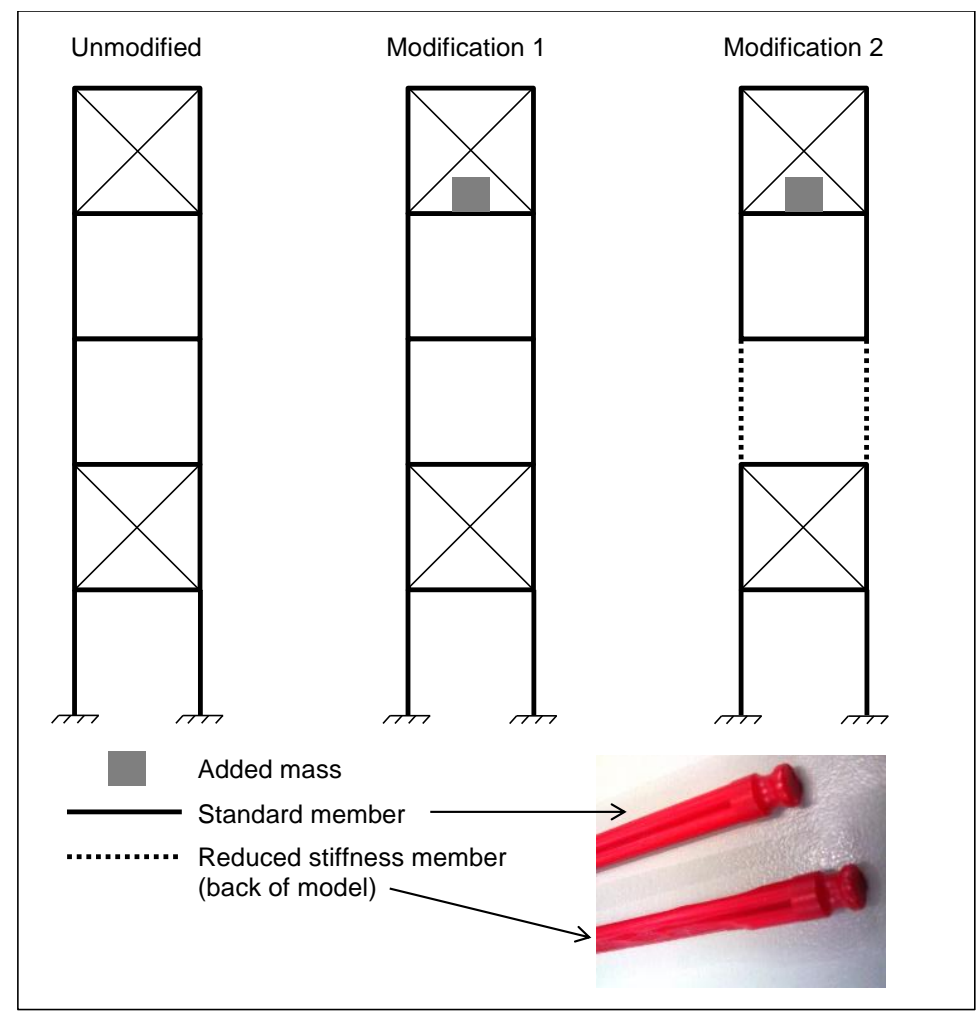

Figure 12: Modifications made to model structure

\section{DISCUSSION}

THIS paper demonstrates the potential for using controlled blurred images to monitor structural tests using off-the-shelf low cost equipment and digital photogrammetry. It may be used for a wide range of applications where imaging 
hardware provides sufficient image and temporal resolution. The proposed solution further extends the capabilities of photogrammetric monitoring by allowing vibration patterns to be identified without the requirement of high imaging speed sensors, which are expensive.

\section{Assessing the accuracy of the system}

An important question is always the accuracy, and this was assessed in another series of simplified tests. Individual targets were mounted directly to the table surface. Since the table motion could be accurately recorded by laser displacement gauge and accelerometers, these can be directly compared with the recorded motion. Various different amplitudes and target sizes were tested, along with different sized circular targets.

Results for errors between laser displacement gauge measurement and the image-based distance measurement are given in figure 13. A standard deviation of $\pm 0.158 \mathrm{~mm}$ was observed, with a mean error of just $-0.115 \mathrm{~mm}$ for a cameraobject distance of $1 \mathrm{~m}$. Using a $95 \%$ confidence interval, the measured distances are considered accurate to within $0.38 \mathrm{~mm}$. In the image space at this scale, this represents 1.43 pixels at the $95 \%$ confidence level. Whilst the accuracy of the current measurement algorithm when expressed in pixels is poorer than conventional target measurement algorithms such as weighted centroid or ellipse fitting, images are obtained at the sensor's highest resolution without having to consider the imaging frequency limitation.

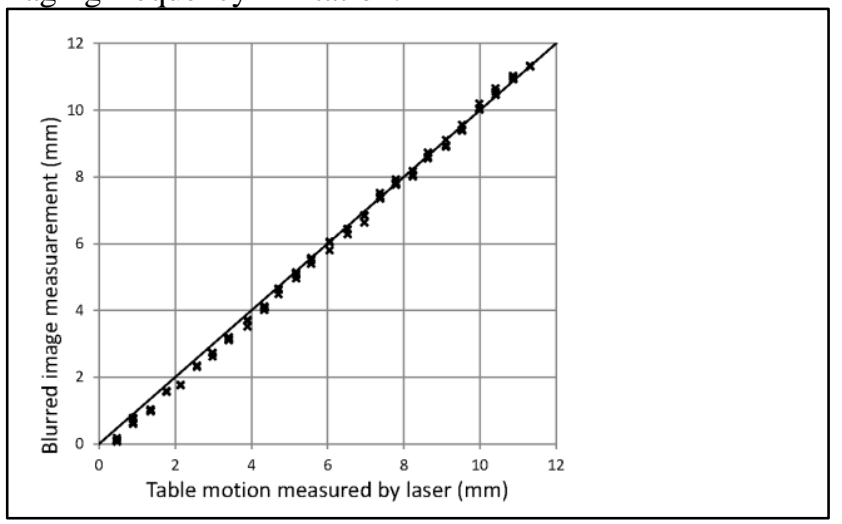

Figure 13: Results of accuracy assessment

The D80 cameras have a sensor resolution of $10.2 \mathrm{MP}$, at which resolution they were found to have a maximum continuous rate of $3 \mathrm{fps}$. These sensors would be limited to recording vibrations of less than $1.5 \mathrm{~Hz}$ at their maximum resolution due to the Nyquist sampling theorem. Utilising the proposed method, these sensors demonstrated the ability to measure the amplitude and shape of the dynamic response of structures at a frequency of $12 \mathrm{~Hz}$. This approach also allows this to be achieved very efficiently, using a minimum number of images and sensors. 
Tests have demonstrated how the approach may be used to identify the changes in the vibration envelope of structures, which in turn may be used to identify structural changes, e.g. the presence of localised damage.

The photogrammetric arrangement makes possible monitoring of displacements to assess static properties of the structure as well as dynamic. Although it is possible to calculate absolute displacements from acceleration measurements by double integration, the result usually exhibits a drift, and alternative sensors are normally necessary for monitoring of static structural properties. The arrangement of image sensors and targets makes possible recording of overall displacements of a structure, for assessment of the structure's static properties.

Imaging sensors have been used for monitoring vibrations in the conventional way by recording displacement-time history, from which frequency information can be identified using the discrete Fourier transform (Choi et al., 2011; Helfrick et al., 2011; Warren et al., 2011). This monitoring requires cameras with higher speeds at the cost of lower image resolutions. It is possible to improve measurement accuracy by 'zooming-in' on a single target, but the advantage of multiple monitoring points would be lost. The advantage of a very high number of monitoring points is of particular interest in the dynamic case. Maintaining a larger image scale allows the higher number of monitoring points, reducing undesirable interpolation.

Control of the image acquisition time and knowledge of the anticipated motion frequency is necessary to ensure that an appropriate shutter speed is selected. A shutter speed too short results in recorded motion that does not confirm to the sinusoidal pattern. In practice, relatively fast motion can be easily measured as the camera speed can be adjusted and the image exposure will remain acceptable. The shutter speed can be extended for slower vibration, but this may result in images being unacceptable overexposed. In case studies, neutral density filters have been used to reduce image exposure, allowing shutter speeds in the order of seconds to be used. Vibrations with a period of a second have been recorded successfully, but possible frequencies are limited by the imaging system.

While many techniques for interpreting dynamic response make use of frequency information, the proposed approach is frequency independent, rather emphasising spatial measurement of the vibration envelope. The acceleration time history from accelerometer data would usually be used for the identification of multiple frequencies in broadband excitation. When used with frequency-selective excitation, direct spatial measurement of the vibration envelope exhibits nodes, antinodes and shape curvature that can be used for dynamic assessment (Abdel Wahab \& De Roeck, 1999). Although only a sinusoidal function has been used in this study to simulate the blur of the target, other functions could be used, depending on the particular regime of motion expected for the structure under observation.

For measurement in 3D, it was recognised that difficulties in target matching exist and how they were overcome has been described. In many static structural testing scenarios, the deformation shape of structure is relatively easy to predict, since the structure usually deforms in the direction of the applied load, and the use of one-dimensional gauges is often suitable. Out-of-plane movement is more 
likely to be encountered during dynamic testing, since excited vibration modes could be longitudinal, transverse or torsional. Therefore, 3D monitoring, such as that presented here, may be desirable in more dynamic testing cases. Triaxial variants of accelerometers are also available, but each sensor is upgraded at added cost and more data-acquisition channels are needed, whilst each individual photogrammetric target is relatively inexpensive.

Accelerometers are more sensitive than the proposed image-based approach. Their high sensitivity and frequency information allows detection of vibrations of very low amplitude and high frequency. When photogrammetry is used for monitoring structural tests it is often commented that traditional contact gauges are known to be more accurate, and are usually used to assess the accuracy of the photogrammetric measurement system (Yoneyama et al., 2005; Ronnholm et al., 2009). However, the cost of an individual accelerometer is high, and extensive cable infrastructure and data monitoring hardware is required. Since individual accelerometers add significant cost to a monitoring system, as well as time to install, fewer sensors are usually possible than the image based approach used here. When determining modal shapes it is desirable to reduce the interpolation necessary between monitoring points by increasing their number (Carden \& Fanning, 2004).

If employed for monitoring outdoors, the approach could clearly be affected by factors such as poor weather. Active targets built from high-intensity L.E.D.s have been discussed by Wahbeh et al. (2003) to allow improved monitoring during night hours and in dull weather. The image processing algorithm used is likely to be successful on this kind of similar images, and this may be an area for future investigation. Other sensors that do not rely on line-of-sight are not without their own difficulties, and Battista (2011) commented on experiencing missing data due to cable infrastructure problems.

\section{CONCLUSION}

THIS paper has presented a novel approach to identifying vibration patterns in civil engineering structures using long-exposure images, in which the targets appear blurred because of the motion of the structure. Photogrammetry has already demonstrated its use for monitoring of structural tests where hardware has sufficient imaging speed. The proposed approach aims to directly record the envelope of sinusoidal vibration, rather than instantaneous deformed shapes. This allows sensors with higher image resolutions to be used and at smaller scale in the field environment. Laboratory tests demonstrate the high quantity of measurements that can be achieved with only a few sensors, and the accuracy of the measurements has been assessed.

The data collected using the blurred-image approach has been compared with the traditional dynamic monitoring instruments. It has also been demonstrated how the approach can be used to detect structural changes in a series of model structures. The new frequency independent approach expands the capabilities of existing sensors which have otherwise had their applications restricted by their imaging frequency. 
MCCARTHY et al. Monitoring 3D Vibrations in Structures Using High Resolution Blurred Imagery

\section{REFERENCES}

AbDel Wahab, M.M. \& De RoecK, G., 1999. Damage Detection in Bridges Using Modal Curvatures: Application To a Real Damage Scenario. Journal of Sound and Vibration, 226(2): 217-235.

Banham, M. \& KatSAGgelos, A.K., 1997. Digital image restoration. Signal Processing Magazine, (March): 24-41.

Battista, N. De, Westgate, R. \& Koo, K., 2011. Wireless monitoring of the longitudinal displacement of the Tamar Suspension Bridge deck under changing environmental conditions. In: M. TOMIZUKA, ed. Sensors and Smart Structures Technologies for Civil, Mechanical, and Aerospace Systems Proceedings of the SPIE: 79811O-15.

BoraCCHI, G., CAGLIOTI, V. \& GiUSTI, A., 2007. Ball position and motion reconstruction from blur in a single perspective image. In: 14th International Conference on Image Analysis and Processing. Modena: IEEE Comput. Soc.

BROWNJOHN, P.J.M.W., 2011. Structural health monitoring: Examples and benefits to structure stakeholders. The Structural Engineer, 89(9): 24-26.

CARDEN, E.P. \& FANNING, P., 2004. Vibration Based Condition Monitoring: A Review. Structural Health Monitoring, 3(4): 355-377.

Chol, H.-S., Cheung, J.-H., KiM, S.-H. \& AHN, J.-H., 2011. Structural Dynamic Displacement Vision System using Digital Image Processing. NDT \& E International, 44(7): 597-608.

CHOPRA, A., 2007. Dynamics of Structures, $3^{\text {nd }}$ Edition, Prentice Hall: Upper Saddle River, NJ (USA).

Clough, R. \& Penzien, J. 2010. Dynamics of Structures, $2^{\text {nd }}$ Edition (Revised), Computers and Structures: Berkeley, CA (USA).

Cross, E.J., KoO, K.Y., BROWNJOHN, J.M.W., \& WORDEN, K., 2013. Long-term monitoring and data analysis of the Tamar Bridge. Mechanical Systems and Signal Processing, 35(1-2): 16-34.

HARVEY, B., 2008. Testing and monitoring of structures: traps for the unwary. Structural Engineer, (October): $22-24$.

Helfrick, M.N., NiEZRECKI, C., AvitABiLE, P. \& SCHMidT, T., 2011. 3D digital image correlation methods for full-field vibration measurement. Mechanical Systems and Signal Processing, 25(3): 917-927.

KASINOS, S., PALMERI, A. \& LOMBARDO, M., 2015. Using the vibration envelope as damage-sensitive feature in composite beam structures, Structures, 1:67-75.

LANGE, J. \& BENNING, W., 2006. Crack detection at concrete construction units from photogrammetric data using image processing procedures. In: N. KERLE \& A. SKIDMORE, eds. Proceedings of the ISPRS Commission VII Symposium "Remote Sensing: From Pixels to Processes". Enschede, The Netherlands.

Luhmann, T., 2011. 3D Imaging - How to Achieve Highest Accuracy. In: F. REMONDinO \& M. R. SHORTIS, eds. Proc. of SPIE: Videometrics, Range Imaging, and Applications XI. Munich, Germany, 808502-1-11.

LUhMANN, T., RoBSON, S., KYle, S., HARLEY, I. 2006. Close range photogrammetry: principles, techniques and applications, Dunbeath: Whittles.

McCARThY, D.M.J., ChandLeR, J.H. \& PALMERI, A., 2013. Monitoring Dynamic Structural Tests Using Image Deblurring Techniques. In: B. BASU, ed. Key Engineering Materials. Dublin, Ireland: Trans Tech Publications, 569-570:932-939.

MCCARThY, D.M.J., Chandler, J.H. \& PALMERI, A., 2014. 3D Case Studies of Monitoring Dynamic Structural Tests using Long Exposure Imagery. International Archives of Photogrammetry, Remote Sensing and Spatial Information Sciences, XL-5, 23-25 June 2014, Riva del Garda, Italy, pp. 407-411.

OLASZEK, P., 1999. Investigation of the dynamic characteristic of bridge structures using a computer vision method. Measurement, 25(3): 227-236.

PALMERI, A. \& LOMBARDO, M., 2011. A new modal correction method for linear structures subjected to deterministic and random loadings, Computers and Structures, 89(11-12):844-854.

Psimoulis, P. A. \& STIROS, S.C., 2008. Experimental Assessment of the Accuracy of GPS and RTS for the Determination of the Parameters of Oscillation of Major Structures. Computer-Aided Civil and Infrastructure Engineering, 23(5): 389-403.

RoberTs, G.W., Meng, X. \& Dodson, A.H., 2004. Integrating a Global Positioning System and Accelerometers to Monitor the Deflection of Bridges. Journal of Surveying Engineering, 130(2): $65-72$. 
MCCARTHY et al. Monitoring 3D Vibrations in Structures Using High Resolution Blurred Imagery

Ronnholm, P., NuikKa, M., SuOminen, A., SAlo, P., HyypPa, H. \& Pontinen, P., 2009. Comparison of measurement techniques and static theory applied to concrete beam deformation. The Photogrammetric Record, 24(128): 351-371.

THOMAS, H. \& CANTRÉ, S., 2009. Applications of low budget photogrammetry in the geotechnical laboratory. The Photogrammetric Record, 24(December): 332-350.

WAHBEH, A.M., CAFFREY, J.P. \& MASRI, S.F., 2003. A vision-based approach for the direct measurement of displacements in vibrating systems. Smart materials and structures, 12: 785-794.

WANG, S., GUAN, B., WANG, G, LI, Q., 2007. Measurement of sinusoidal vibration from motion blurred images. Pattern Recognition Letters, 28(9):1029-1040.

WarRen, C., NieZReCKi, C., AVitabile, P., Pingle, P., 2011. Comparison of FRF measurements and mode shapes determined using optically image based, laser, and accelerometer measurements. Mechanical Systems and Signal Processing, 25(6): 2191-2202.

YITZHAKY, Y. \& BOSHUSHA, G., 2000. Restoration of an image degraded by vibrations using only a single frame. Optical Engineering, 39(8):2083-2091.

Yoneyama, S., KitAgwa, A., KitamURA, K., KiKUTA, H., 2005. Deflection distribution measurement of steel structure using digital image correlation. In: L. M. Hanssen \& P. V. Farrell, eds. Optical Diagnostics, 58800G 1-8.

Résumé

Zusammenfassung 